\title{
Real-Time Economic Frequency Regulation for Partitioned Microgrids Based on Cost-Driven Droop Function
}

\author{
Qian Hu, Student Member, IEEE and Siqi Bu, Senior Member, IEEE
}

\begin{abstract}
With the increase of the number and geographic dispersion of distributed energy resources (DERs), the microgrid (MG) has become scattered along with the higher complexity of control and communication. This paper proposes a two-level regulation strategy to achieve the local objective of frequency regulation and power sharing as well as the global objective of economic operation in the real time for the scattered MG. Firstly, the MG integrated with multiple DERs is partitioned into several nanogrids (NGs) such that DERs connecting on the same feeder are grouped in the same NG for the efficient and cost-effective communication and control. Then in the NG level, the total power mismatch of the entire MG can be learned and an optimal incremental cost can be agreed by each NG through the fastest distributed linear averaging (FDLA) and discrete-consensus algorithm, respectively. In the DER level, a cost-driven droop gain is developed to indicate the willingness of each DER in the NG to participate into the frequency regulation service. The pinning-based protocol is formulated to regulate the frequency and meanwhile enable the proportional power sharing among DERs based on the economic droop function. Case studies satisfactorily demonstrate the effectiveness of the proposed regulation strategy for the economic frequency regulation in the tested MG.
\end{abstract}

Index Terms-- economic frequency regulation, ancillary service participation factor, nanogird, pinning control, discreteconsensus, fastest distributed linear averaging (FDLA)

\section{INTRODUCTION}

$\mathrm{T}$ he popularity of microgrids (MGs) has increased due to a flexible and effective integration of distributed energy resources (DERs) into power systems [1]. Particularly, the scattered MG, which is integrated with a large number of geographically dispersed DERs, requires high-speed and reliable computation and communication capabilities. To improve the reliability and reduce the communication burden, the scattered MG can be divided into several nanogirds (NGs) with respective NG controllers [2]-[4]. Power support is enabled for the local loads through the communication among NGs when the scattered MG is disconnected from the main grid. However, it is more challenging to maintain a reliable operation in the islanded mode [5]: On the one hand, severe frequency deviations might appear due to the low-inertia characteristic of the MG; On the other hand, the critical demand-supply equilibrium must be maintained which requests an accurate load sharing mechanism to compensate the sudden active power mismatches.

To address the aforementioned challenges, the conventional hierarchical control structure including inner control, primary (droop) control and secondary control is implemented [6]-[9]. Through current and voltage control loop, inner control provides high frequency switching pulses for the power electronics-interface of the DER to produce the sinusoidal voltage with the desired frequency. Droop-based primary control aims to locally provide the power sharing among DERs and adjust the voltage and frequency reference provided to the inner control. Secondary control is to compensate the voltage and frequency deviations via centralized or decentralized communications. Such the common control architecture is able to provide reliable frequency and voltage regulation but lack of the consideration from the perspective of economics.

Conventional economic dispatch problem for the power system is mainly solved in centralized way which depends on a central system operator to collect the information from all units and conduct the computation procedure [10]. Such the centralized economic operation is adapted in a higher layer control so called tertiary control for MGs to achieve the economy-effectiveness. But it more targets at the off-line operation among multiple MGs [5]. Considering the increasing complexity due to the growing integration of DERs and flexible loads, the centralized operation cannot be compatible with the distributed nature of DERs in the MG. Consequently, decentralized algorithms have been used in solving economic dispatch problems in some studies, where consensus-based algorithm is a popular approach [11][12]. However, most of the studies implementing distributed algorithms focus on the off-line optimal operation, which are not desired for the real-time coordination to meet the flexibility of the MG.

In most of the existing studies for the economic power sharing in the MG [4][13][14], loads are proportionally supplied by DERs according to their capacity ratings in a prespecified manner. In fact, frequency regulation by adjusting power provision is one of ancillary services which can be carried out with the consideration of appropriate incentives [15]. An economic power sharing should incorporate the individual participation willingness of each DER for the regulation ancillary service. Hence, the design for a costeffective droop function is highly required to enhance the optimal performance of the frequency control and the power sharing in terms of the economy-effectiveness.

Therefore, based on the aforementioned, in this paper, a coordinated two-level regulation strategy is proposed for the real-time economic frequency regulation considering the participation willingness of each DER for the regulation service. The entire operation only relies on local measurements based on the peer-to-peer communication links. The contributions of this paper are highlighted below:

1. The MG is partitioned into several nanogrids (NGs) with assigned DER leader, which offers a cost-effective and 
efficient control option for the MG. The communication burden can be reduced significantly.

2. In the upper NG level, two distributed algorithms: FDLA and discrete consensus algorithm, are implemented so that the total power mismatch in the MG and the optimal incremental cost can be fast sensed and updated by each $\mathrm{NG}$ without the need of the central controller.

3. Ancillary service participation factor is proposed to indicate the willingness of the DER to participate into the frequency regulation service based on the incremental cost. In the lower DER level, an economic-oriented droop function which integrates the participation factor provides a cost-driven mechanism for the load power sharing.

4. The proposed pinning-based protocol enables the frequency synchronization to the reference value and the incentivized power provision of DERs via neighboring communication links.

\section{DER CONTROL STRUCTURE AND MG PARTITION}

The DER unit supplied by a DC source is integrated into the MG through DC/AC inverter, LC filter and output connector, which is shown Fig.1. A hierarchical control including the inner control, droop-based primary control and secondary control is applied to the DER unit to regulate the output voltage and frequency within the desired range. As economic power sharing and frequency regulation is of our interest in this paper, the inner control with faster dynamics is neglected. We consider the frequency droop-based primary control for the DER:

$$
\omega_{i}=\omega_{i}^{*}+m_{i} P_{i}
$$

where $\omega_{i}$ is the angular frequency reference provided to the inner control loop; $\omega_{i}^{*}$ is the nominal angular frequency; $m_{i}$ is the frequency droop gain which is configured based on the power rating of the DER; $P_{i}$ is the output active power. The frequency deviation resulted from the primary control is compensated by the secondary control, which requires the decentralized communication with neighboring DERs.

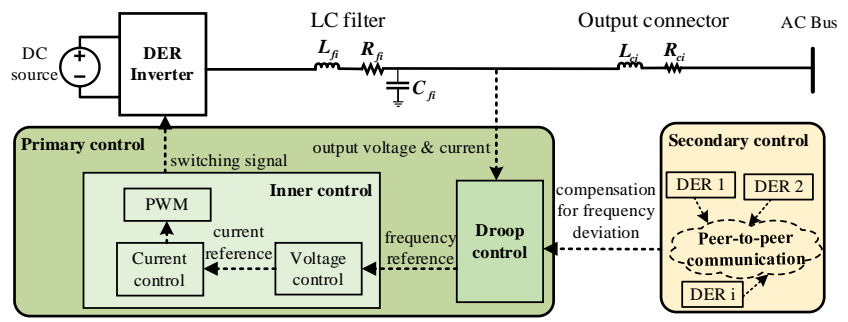

Fig. 1. DER unit with hierarchical control for the frequency regulation

In fact, the control complexity and the communication burden increase with the number and geographic dispersion of DERs in the MG. For the sake of an efficient and costeffective cooperation among DERs, it is essential to divide the MG into several NGs to conduct the relevant control actions. Fig.2 shows a schematic diagram for the MG partition which is applied in the paper.

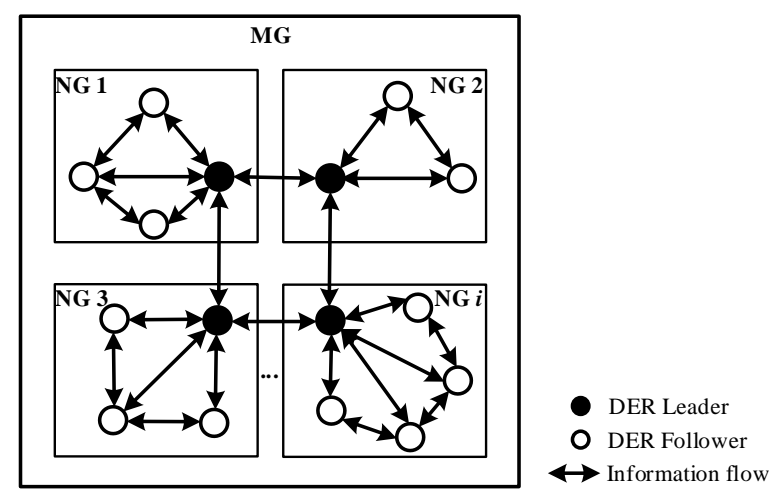

Fig. 2. The MG is partitioned into multiple NGs for an efficient control operation with reduced communicaton burden

In practice, the partition of the MG can be conducted in different ways considering various factors, such as the specific category of DERs and the relative geographic locations of DERs. In this paper, DERs connecting on the same distribution feeder are organized into the same NG. At least one DER is selected as the leader and other DERs are referred as the followers in the NG. In the level of the NG, each NG works collaboratively as an independent entity with other NGs in the MG. As the representative of the NG, the DER leader can exchange the local information with other neighboring leaders via peer-to-peer communication links. In the level of the DER, every single DER communicate with neighboring DERs belonging to the same NG. In comparison with the nonpartitioned MG, less communication and controllers are needed for the partitioned one. Therefore, such the partition along with the corresponding communication network could significantly save the communication bandwidth and the control cost.

\section{Proposed Two-Level Regulation Strategy}

A two-level regulation strategy including the NG-level and the DER-level is developed to adapt to the partitioned MG. The objective of the upper NG-level is to ensure the economic operation of the MG by coordinating the incremental cost of all NGs. The objective of the lower DER- level is to maintain the frequency and ensure an economic power sharing among DERs. Main functions of each level are presented in Fig.3.

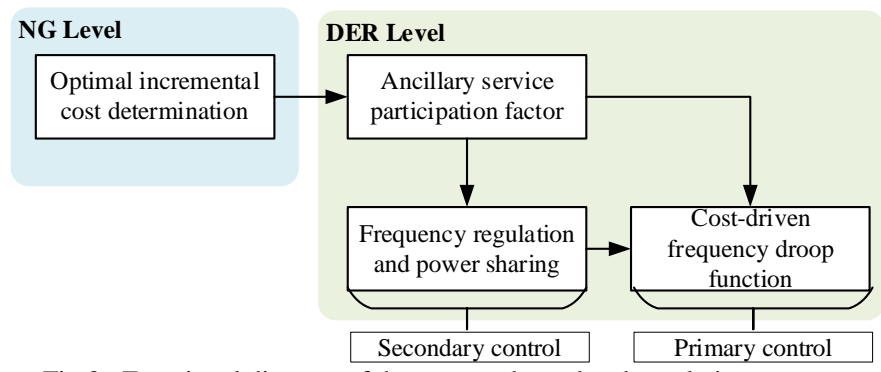

Fig.3. Functional diagram of the proposed two-level regulation strategy

If there are sudden changes of the power demand occur in any NGs, the total power mismatch of the MG can be fast learned by each NG by means of the FDLA technique. An optimal incremental cost subsequently can be achieved by each NG through the incremental cost consensus algorithm, which is the input signal to the lower level. The pinning DER in the lower DER-level is able to receive the output signal from the upper 


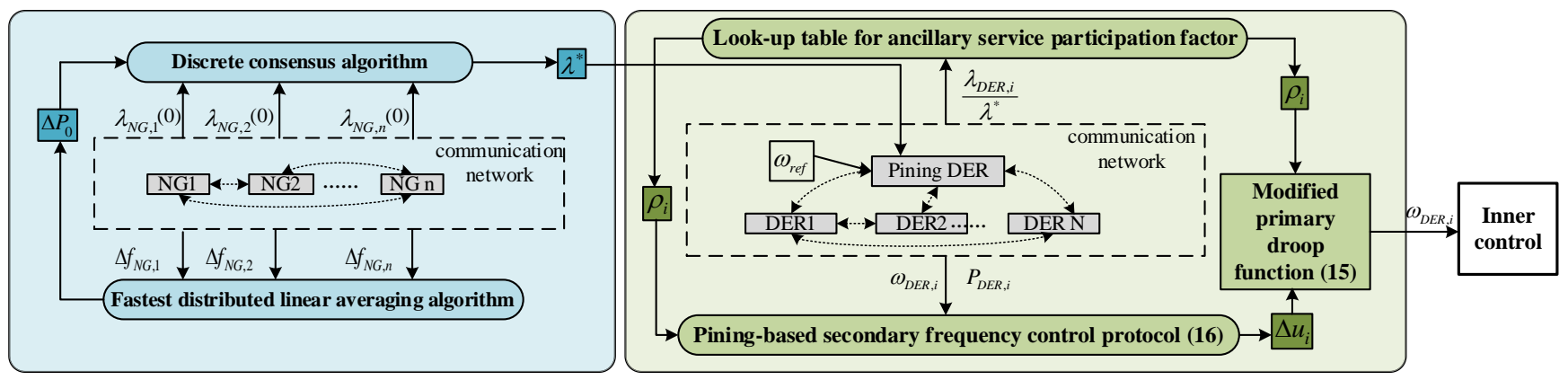

Fig. 4. Schematic diagram of the proposed two-level regulation strategy

level and access the reference frequency signal. Optimal incremental cost developed from the upper level is shared with the rest of DERs in the NG by the DER leader. Then the follower DERs will determine the ancillary service participation factor by comparing the optimal incremental cost signal with its initial incremental cost. Such the factor can be embedded into the frequency droop gain to formulate a costdriven droop function. Based on the developed pinned control protocols, the frequency can be regulated to the reference value and meanwhile the proper power sharing considering the ancillary service participation factor can be acquired. The design principle of the two-level regulation strategy is shown in Fig.4. Operation details of each level are discussed in the following subsections.

\section{A. Upper NG-level}

Assuming the total number of the NGs in the MG is $n=$ $1,2, \ldots, n$, to achieve a cost-effective operation of the MG, the economic dispatch problem is utilized to minimize the total generation cost, which is formulated as:

$$
\begin{array}{lc} 
& \min \sum_{i=1}^{n} C_{i}\left(P_{N G, i}\right) \\
\text { s.t. } & P_{D}-\sum_{i=1}^{n} P_{N G, i}=0 \\
& P_{N G, i}^{\min } \leq P_{N G, i} \leq P_{N G, i}^{\max }
\end{array}
$$

where $P_{N G, i}$ denotes the output power of $i^{t h} \mathrm{NG}$ and $P_{D}$ denotes the total power consumption in the MG including network losses. $P_{N G, i}^{\min }$ and $P_{N G, i}^{\max }$ is the lower and upper capacity bound of $i^{\text {th }} \mathrm{NG}$, respectively. The quadratic form $C_{i}\left(P_{N G, i}\right)=a_{i} P_{N G, i}{ }^{2}+b_{i} P_{N G, i}+c_{i}$ is used as the generation cost function for the DER leader of $i^{\text {th }}$ NG. The incremental cost of the DER leader is:

$$
\lambda_{N G, i}=\frac{\partial C_{i}\left(P_{N G, i}\right)}{P_{N G, i}}=2 a_{i} P_{N G, i}+b_{i}
$$

During the steady-state operation, all NGs can maintain at the same operating frequency $f_{s s}$. By adding individual droop function (1) of each DER leader together, the equivalent droop function of the entire MG can be represented by [4]:

$$
\sum_{i=1}^{n} \frac{1}{m_{N G, i}} \Delta f_{N G, i}=\sum_{i=1}^{n} \Delta P_{N G, i}
$$

where $\Delta f_{N G, i}=f^{*}-f_{N G, i}$ denotes the operating frequency deviation from the nominal frequency for the DER leader. Then the total power imbalance in the MG can be represented by the local measurement of the frequency deviation and the local droop gain for each DER leader:

$$
\sum_{i=1}^{n} \frac{1}{m_{N G, i}} \Delta f_{N G, i}=P_{D}-\sum_{i=1}^{n} P_{N G, i}=\Delta P
$$

Once the operating condition varies at a certain time, the power imbalance occurs in the MG, which is referred as the initial power imbalance $\Delta P_{0}$. It should be noted $\Delta P_{0}$ is a global information. Differently from the conventional economic dispatch problem which requires the offline prediction of the power consumption, $P_{D}$ here is the actual power demand which should be met by the online cooperative operation of NGs. Hence, a fast-online distributed approach should be developed to acquire $\Delta P_{0}$ by means of the local information exchanging. In this connection, the fastest distributed linear averaging (FDLA) algorithm is introduced to locally estimate $\Delta P[16]:$

$$
y_{i}(k+1)=y_{i}(k)+\sum_{j=1}^{n} w_{i j} y_{j}(k)
$$

where $k$ is the discrete step index which has $k=0,1,2 \ldots$; $y_{i}(k)$ is the local state of $i^{t h} \mathrm{NG}$ at step of $k . w_{i j}$ is the entry of the weight matrix $W$, which describes the weight on the communication link from $i^{\text {th }} \mathrm{NG}$ to $j^{\text {th }} \mathrm{NG}$. After iterations, state of each node will fast converge to a common value:

$$
\lim _{k \rightarrow \infty} y_{i}(k)=\frac{1}{n} \sum_{j=1}^{n} y_{i}(0)
$$

Equation (9) indicates that the state evolution by (8) will finally lead each NG approach to the average value of initial states. The weight matrix $W$ is properly designed to minimize the convergence time of the distributed linear averaging algorithm, as follows:

$$
\begin{gathered}
\min \left\|W-(1 / n) 11^{T}\right\|_{2} \\
\text { s.t. } W \in \mathcal{S}, 1^{T} W=1^{T}, W 1=1
\end{gathered}
$$

where $\quad \mathcal{S}=\left\{W \in R^{n \times n} \mid w_{i j}=0\right.$ if $\{i, j\} \notin \mathcal{E}$ and $\left.i \neq j\right\}, \quad 1$ represents the vector with all entries equal to one. The constraints are developed based on the convergence conditions for (8) [16]. Once the power demand is changed, the FDLA algorithm is adapted so that the estimation of $\Delta P_{0}$ can be accessed by each NG. The detailed procedure is presented in Algorithm 1. 


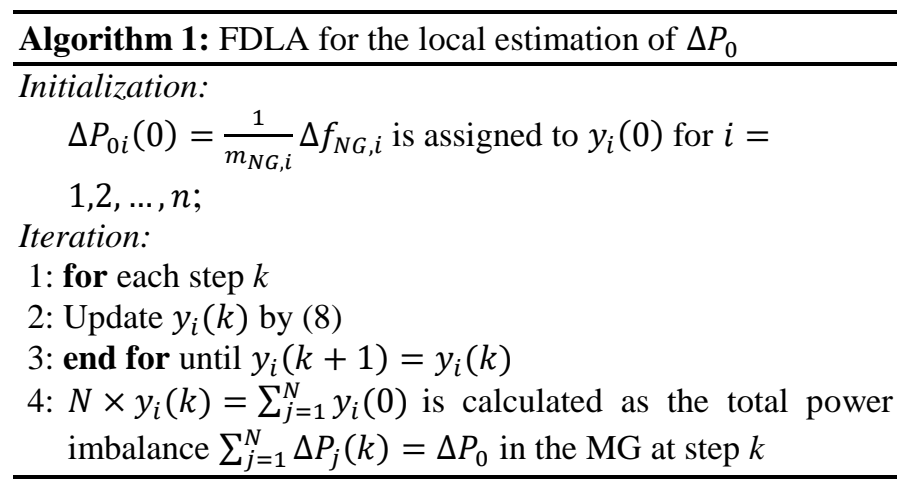

Once the information of initial power imbalance $\Delta P_{0}$ is available for the DER leader of each NG, all DER leaders coordinately schedule the power dispatch of its NG to meet the new power demand in an economic manner. Regarding this matter, the equal increment cost principle of economic dispatch, i.e., $\lambda_{N G, 1}=\lambda_{N G, 2}=\ldots=\lambda_{N G, n}=\lambda^{*}$, should be satisfied for each participating NG. The discrete consensus algorithm is applied to achieve the unified incremental cost [17]:

$$
\lambda_{N G, i}(k+1)=\sum_{j=1}^{n} \frac{l_{i j}}{\sum_{j=1}^{n}\left|l_{i j}\right|} \lambda_{N G, j}(k)+\mu \Delta P(k)
$$

where $\mu>0$ can be referred as the convergence coefficient as its value influences the convergence rate of the algorithm. $l_{i j}$ is the entry of Laplacian matrix $L$ of the communication network, defined as:

$$
l_{i j}=\left\{\begin{array}{c}
-1,\{i, j\} \in \mathcal{E} \\
d_{i}, i=j \\
0, \text { otherwise }
\end{array}\right.
$$

where set $\mathcal{E}$ contains the communication links between $i^{\text {th }} \mathrm{NG}$ and $j^{\text {th }} \mathrm{NG} ; d_{i}$ denotes the number of neighboring NG of $i^{\text {th }}$ NG. If $\Delta P_{0}>0$, which means the power consumption is larger than the power output in the MG and thus more power should be generated, the current incremental cost of each NG should be increased. The incremental cost is updated by (12) through the information exchange with its neighbors considering the limitation of the generation capacity. The detailed algorithmic process is shown in the following.

Algorithm 2: Discrete Consensus Algorithm to Locally Find Optimal $\lambda^{*}$

\section{Initialization:}

Set unified $\lambda_{N G, i}(0)$ and compute $P_{N G, i}(0)$ based on the generation cost function for $i=1,2, \ldots, n ; \Delta P_{0}$ is assigned to $\Delta P(0)$

\section{Iteration:}

1: for each step $k$

2: Update $\lambda_{N G, i}(k)$ by (12)

3: Compute $P_{N G, i}(k)$ according to (5)

4: $\quad$ if $P_{N G, i}^{\min } \leq P_{N G, i} \leq P_{N G, i}^{\max }$, calculate $\Delta P(k)$

5: else set $P_{N G, i}=P_{N G, i}^{\min }$ or $P_{N G, i}=P_{N G, i}^{\max } \&$ calculate $\Delta P(k)$

6: end if

7: end for until $|\Delta P(k)|=$ tolerance

8: $\lambda^{*} \leftarrow \lambda_{N G, i}(k), P_{N G, i} \leftarrow P_{N G, i}(k)$

\section{A. Lower DER-Level}

Assuming the total number of the DERs in a NG is $N=$ $1,2, \ldots, N$. Load active power is shared among DERs according to their droop gains to prevent the overloading of DERs. Such the conventional droop control ensures the fair power sharing in agreement with the rating of each DER, yet fails to guarantee the economic operation of the MG. Hence, an economic-oriented droop control should be developed. Firstly, we introduce a parameter $\rho_{i}$ called ancillary service participation factor to indicate the willingness of $i^{\text {th }} \mathrm{DER}$ in the NG to participate in the frequency regulation ancillary service, as follows:

$$
\begin{cases}\rho_{i}<1, & \lambda_{D E R, i}>\lambda^{*} \\ \rho_{i}=1, & \lambda_{D E R, i}=\lambda^{*} \\ \rho_{i}>1, & \lambda_{D E R, i}<\lambda^{*}\end{cases}
$$

where $\lambda_{D E R, i}$ denotes the default incremental cost of $i^{\text {th }}$ DER. If the incremental cost is smaller than the optimal one acquired from the upper level, the DER will have an inventive to provide more power, i.e., larger $\rho_{i}$, and vice versa. The default value of $\rho_{i}$ is determined based on the rule shown in the Table I. The DER will not participate in the ancillary service when $0<\lambda_{D E R, i} / \lambda^{*}<0.25$.

TABLE I. DESIGN RULE of ANCILLARY SERvice PARTICIPATION FACTOR

\begin{tabular}{c|l|l|l|l|l}
\hline \hline$\lambda^{*} / \lambda_{\text {DER,i }}$ & {$[0,0.25)$} & {$[0.25,0.5)$} & {$[0.5,0.75)$} & {$[0.75,1)$} & 1 \\
\hline$\rho_{i}$ & NA & 0.25 & 0.5 & 0.75 & 1 \\
\hline$\lambda^{*} / \lambda_{D E R, i}$ & $(1,1.25]$ & $(1.25,1.5]$ & $(1.5,1.75)$ & $(1.75,2]$ & $>2$ \\
\hline$\rho_{i}$ & 1.25 & 1.5 & 1.75 & 2 & 2 \\
\hline \hline
\end{tabular}

Based on the ancillary service participation factor $\rho_{i}$, an economically optimal droop function can be formulated as:

$$
\omega_{D E R, i}=\omega^{*}+\frac{m_{D E R, i}}{\rho_{i}} P_{D E R, i}+\Delta u_{i}
$$

where $\Delta u_{i}$ is the output from secondary control for the compensation of the frequency deviation. In order to track the frequency reference $\omega_{\text {ref }}$ and achieve the economic power sharing, a pinning-based protocol is developed for secondary frequency control:

$$
\begin{gathered}
\Delta \dot{u}_{\imath}=-c\left(\sum_{j=1, j \neq i}^{N} a_{i j}\left(\omega_{D E R, i}-\omega_{D E R, j}\right)+p_{i}\left(\omega_{D E R, i}-\right.\right. \\
\left.\left.\omega_{r e f}\right)\right)-c \sum_{j=1, j \neq i}^{N} a_{i j}\left(\frac{m_{D E R, i}}{\rho_{i}} P_{D E R, i}-\frac{m_{D E R, j}}{\rho_{j}} P_{D E R, j}\right)
\end{gathered}
$$

where $c$ is the control gain; $A=\left[a_{i j}\right]$ is the adjacency matrix of the corresponding communication network, where $a_{i j}=1$ if $i^{\text {th }}$ DER can receive the information from $j^{\text {th }}$ DER otherwise $a_{i j}=0 ; p_{i}>0$ is the gaining gain if $i^{t h}$ DER is pinned, otherwise $p_{i}=0$.

Denoting $e_{i}=\omega_{i}-\omega_{\text {ref }}$ as the frequency synchronization error of the $i^{t h}$ DER, the error dynamics can be formulated as:

$$
\dot{e}_{i}=\sum_{j=1, j \neq i}^{N} c a_{i j}\left(e_{j}-e_{i}\right)-p_{i} e_{i}
$$

To obtain the stability properties of the proposed pinning synchronization tracking protocols, the Lyapunov candidate shown below can be used.

$$
V_{L Y}=\frac{1}{2} \sum_{i}^{n} e_{i}^{T} e_{i}
$$

Then, by differentiating (18) we can derive that 


$$
\begin{gathered}
\dot{V}_{L Y}=\sum_{i}^{n} e_{i}^{T} e_{i} \\
=\sum_{i}^{n} e_{i}^{T}\left\{\sum_{j=1, j \neq i}^{N} c a_{i j}\left(e_{j}-e_{i}\right)-p_{i} e_{i}\right\} \\
\leq \sum_{i}^{n} e_{i}^{T} \sum_{j=1, j \neq i}^{N} c a_{i j}\left(e_{j}-e_{i}\right)-\sum_{i}^{n} p_{i}\left\|e_{i}\right\|^{2} \\
\leq \sum_{i}^{n}\left\|e_{i}^{T}\right\| \sum_{j=1, j \neq i}^{N} c a_{i j}\left\|e_{j}-e_{i}\right\|-\sum_{i}^{n} p_{i}\left\|e_{i}\right\|^{2} \\
\leq \sum_{i}^{n}\left\|e_{i}^{T}\right\| \sum_{j=1, j \neq i}^{N} c a_{i j}\left(\left\|e_{j}\right\|+\left\|e_{i}\right\|\right)-\sum_{i}^{n} p_{i}\left\|e_{i}\right\|^{2} \\
=|\varepsilon|^{T}(B-P)|\varepsilon|
\end{gathered}
$$

where $\varepsilon=\left[e_{1}, e_{2}, \ldots, e_{N}\right]^{T}$ denotes the error vector; $B=$ $\left[c a_{i j}\right]$ denotes the coupling configuration of the communication network; $P=\operatorname{diag}\left(p_{1}, p_{2}, \ldots, p_{N}\right)$ denotes the pinning gain matrix. The asymptotic stability of proposed pinning-based secondary control can be achieved if matrix $B-P$ is a Hurwitz matrix, i.e., all its eigenvalues have a strictly negative real part. Given the invariant coupling configuration of the communication network, by designing the proper pinning matrix $P, \lim _{t \rightarrow \infty}|\varepsilon|$ could approach to zero. Furthermore, it can be concluded, given the appropriate pinning gain, the frequency can be restored to the desired state.

Remarks: The previous analysis has shown the controllability of the proposed protocols. As a matter of fact, the pinning controllability can be optimized to improve he control performance. Let $\mathcal{J}(B-P)$ represents the real part of maximum eigenvalues of matrix $(B-P)$ and the pinning controllability improves as $\mathcal{J}(B-P)$ become smaller, i.e., moving further towards to the left half plane [18]. Generally, two factors contribute to the pinning control performance: the selection of the pinning DER and the determination of the pinning gain. There exists a trade-off between the control cost and control efficiency in terms of the selection of pinned nodes. Obviously, pinning all the nodes with highest possible pinning gains would be a trivial solution, considering no constraints on the pinning set and upper bound of the pinning gain $\bar{p}$. However, it is unrealistic for the real applications as not all the nodes are accessible and the control cost would increase significantly. To improve the controllability, one optimization problem can be formulated to minimize $\mathcal{J}(B-$ $P$ ) with the consideration of the constraint on the number of the pining DER and the control cost by determining the pinning gain [19] and the pinning DER [20].

\section{CASE Studies}

The test MG with four NGs is used to verify the effectiveness of the proposed two-level regulation strategy in MATLAB/Simulink. The schematic diagram of the system and the corresponding communication network is shown in Fig.5. For simplicity, it is assumed that each NG contains four DERs and one local variable load. DER1 is set as the pinning DER in each NG which is able to access the desired reference value. The physical configurations of the DER are set based on the specifications presented in [21]. The generation cost parameters and initial power generation of each NG based on the unified initial incremental cost $\lambda^{*}(0)=8.9 \$ / \mathrm{kWh}$ are given in Table II.

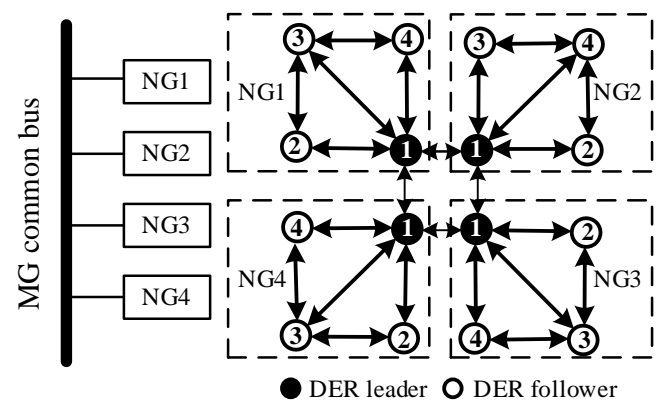

Fig. 5. Schematic diagram of the test MG (left) and the communicaiton network of the NG (right)

TABLE II. PARAMETERS OF DER LEADER IN THE NG

\begin{tabular}{l|l|l|l|l}
\hline \hline & $a_{i}$ & $b_{i}$ & $c_{i}$ & $P_{N G, i}(0)(\mathrm{Kw})$ \\
\hline NG 1 & 0.002 & 8 & 20 & 225 \\
\hline NG 2 & 0.004 & 6.5 & 50 & 300 \\
\hline NG 3 & 0.005 & 7.8 & 30 & 110 \\
\hline NG 4 & 0.003 & 5.7 & 40 & 533 \\
\hline \hline
\end{tabular}

\section{B. Coordinated operation in the NG-level}

The MG is operated in the steady-state in the first three seconds with the optimal incremental cost $8.9 \$ / \mathrm{kWh}$, where $P_{D}=\sum_{i=1}^{4} P_{N G, i}=1170 \mathrm{Kw}$. At $\mathrm{t}=3 \mathrm{~s}$, additional $450 \mathrm{Kw}$ and $300 \mathrm{Kw}$ load is added into the NG1 and NG2, respectively, while $120 \mathrm{Kw}$ demand is decreased in the NG3, which makes total power mismatch in the MG is $630 \mathrm{Kw}$. Such a global signal is locally acquired by each NG by applying Algorithm 1 in the NG-level. Weight matrix $W$ that ensures the fastest convergence of the distributed linear averaging iteration is determined by (10) and (11). Fig. 6 shows that the faster convergence can be achieved by FDLA with optimal weighted communication network while for the communication network without optimization the convergence time is longer. The agreement of $157.5 \mathrm{Kw}$ is reached that is the average value of the initial power mismatch in all NGs. Therefore, $\Delta P_{0}$ $=157.5 \mathrm{Kw} \times 4=630 \mathrm{Kw}$ is locally available to each NG, which is consistent with the total power imbalance in the MG. At $\mathrm{t}=7 \mathrm{~s}$, the power consumption in the NG1 is decreased by $300 \mathrm{Kw}$.
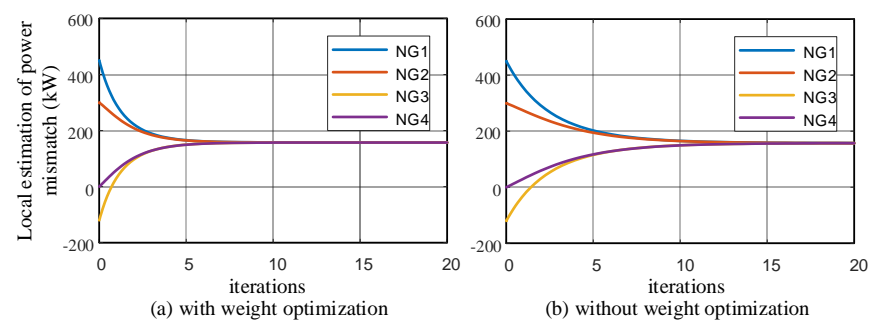

Fig. 6. Convergence performance for the estimation of power mismatch

Similar process for the total power mismatch is conducted again. Every time the demand varies in the MG, the optimal incremental cost $\lambda^{*}$ is coordinately updated by Algorithm 2 
based on the knowledge on $\Delta P_{0}$, which is shown in Fig.7. Here the convergence coefficient $\mu$ is set as $4 \times 10^{-4}$ for the Algorithm 2.

The increase/decrease of the total power demand in the system requires more/less generation and thus the current incremental cost is increased/decreased accordingly for an economic operation. We can observe from Fig.7 that the optimal incremental cost is converged to $9.8 \$ / \mathrm{kWh}$ after $0.3 \mathrm{~s}$ and then down to $9.4 \$ \mathrm{kWh}$. Fig.8 plots the power dispatch that is required to meet the load demand based on the generation cost functions of each NG.

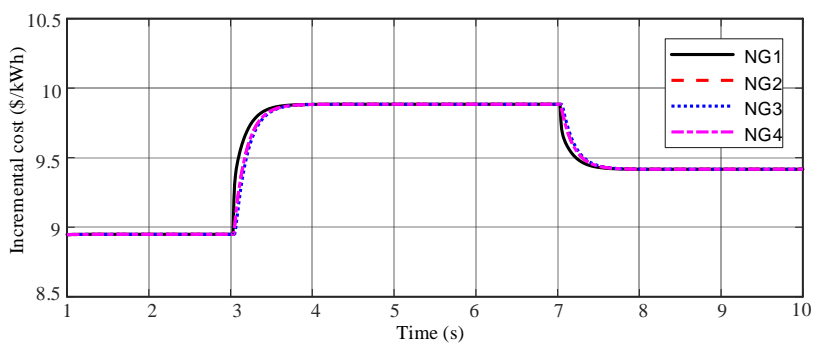

Fig. 7. Optimal incremental cost for the economic opeartion of the MG

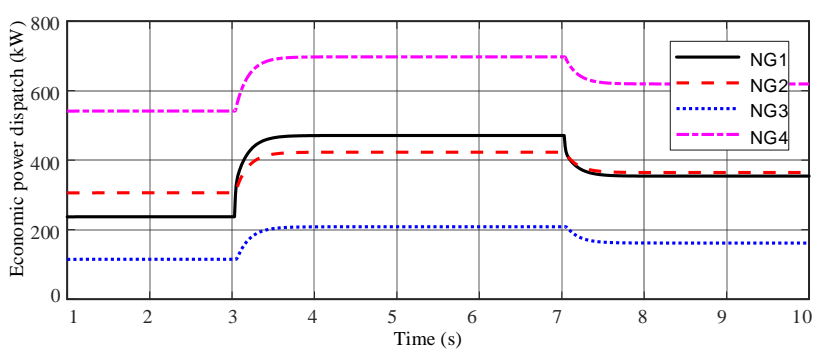

Fig. 8. Economic power disptach from each NG in the MG

Once the optimal incremental $\operatorname{cost} \lambda^{*}$ is found, each leader broadcasts $\lambda^{*}$ to the follower by the neighboring communications. Here we take NG2 as an example to demonstrate the effectiveness of the frequency regulation and economic power sharing in the DER-level. The angular frequency reference $\omega_{\text {ref }}$ is set to $314 \mathrm{rad} / \mathrm{s}(50 \mathrm{~Hz})$ which is provided to the pinning DER in each NG. Control gain $c$ and pinning gain $p_{i}$ is set to 100 and 10 , respectively. The default incremental cost of DER1 to DER4 in the NG2 is [5.2, 7.2, $7.4,20] \$ / \mathrm{kWh}$ and the corresponding ancillary service participation factor is $[2,1.5,1.5,0.25]$, which is acquired based on the Table I. Fig.9 shows the frequency of each DER in the NG2. It is observed that the frequency can be fast restored to the specific reference value within 1 second under the action of the pinning secondary control. As DER1 is pinned, it experiences the smallest frequency drop/increase.

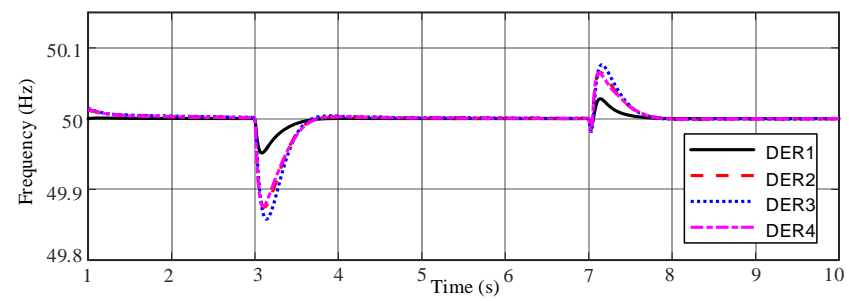

Fig. 9. Frequncy control based on the secondary pinning control protocol
The economic power sharing among DERs is shown in Fig.10. The power output is proportionally to the participation factor of each DER, where DER1 generates the major amount of power due to its largest participation factor where the DER3 has the smallest power output as it has lowest participation factor resulted in the highest default incremental cost.

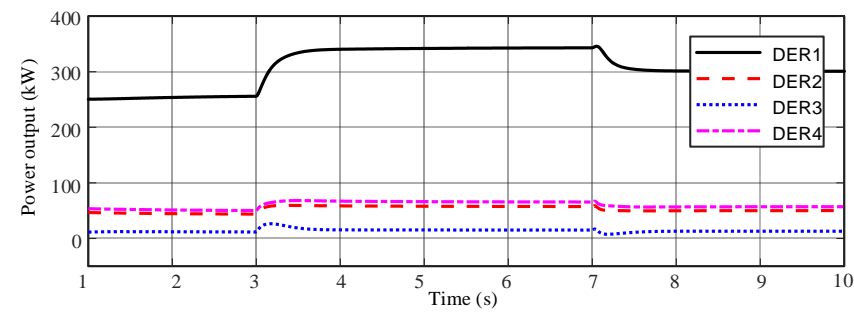

Fig. 10. Economic power sharing among DERs in the NG2

\section{CONCLUSION}

The economic dispatch and the secondary frequency control are incorporated into the proposed two-level regulation strategy for the real-time economic frequency regulation in islanded MGs. The MG is partitioned into several NGs for the cost-effective control, which facilities the flexibility and scalability of the MG and reduce the communication cost. In the NG level, the information sharing among appointed leaders in NGs enables the real-time economic dispatch of the MG. Based on the application of two distributed algorithms (FDLA and consensus algorithm), the optimal incremental cost is updated only if the power imbalance occurs in the MG. In the DER level, pining-based secondary frequency control protocol is developed to regulate the frequency and ensure the economic power sharing among DERs. The cost-driven droop function integrated with ancillary service participation factor is formulated which is beneficial for building an effective and efficient ancillary service market. The simulation results on the test MG have shown that frequency can be fast regulated and the economic power sharing can be achieved effectively.

\section{REFERENCES}

[1] R. H. Lasseter et al., "CERTS Microgrid Laboratory Test Bed," IEEE Trans. Power Deliv., vol. 26, no. 1, pp. 325-332, Jan. 2011.

[2] A. Werth, N. Kitamura, and K. Tanaka, "Conceptual Study for Open Energy Systems: Distributed Energy Network Using Interconnected DC Nanogrids," IEEE Trans. Smart Grid, vol. 6, no. 4, pp. 1621-1630, 2015.

[3] M. Rafiee Sandgani and S. Sirouspour, "Energy Management in a Network of Grid-Connected Microgrids/Nanogrids Using Compromise Programming," IEEE Trans. Smart Grid, vol. 9, no. 3, pp. 2180-2191, 2018.

[4] Q. Zhou, M. Shahidehpour, Z. Li, L. Che, A. Alabdulwahab, and A. Abusorrah, "Compartmentalization Strategy for the Optimal Economic Operation of a Hybrid AC/DC Microgrid," IEEE Trans. Power Syst., vol. 35, no. 2, pp. 1-1, 2019.

[5] D. E. Olivares et al., "Trends in microgrid control," IEEE Trans. Smart Grid, vol. 5, no. 4, pp. 1905-1919, 2014.

[6] J. M. Guerrero, J. C. Vasquez, J. Matas, L. G. De Vicuña, and M. Castilla, "Hierarchical control of droop-controlled AC and DC microgrids - A general approach toward standardization," IEEE Trans. Ind. Electron., vol. 58, no. 1, pp. 158-172, 2011.

[7] A. Bidram and A. Davoudi, "Hierarchical structure of microgrids control system,” IEEE Trans. Smart Grid, vol. 3, no. 4, pp. 1963-1976, 2012.

[8] A. Mehrizi-Sani and R. Iravani, "Potential-function based control of a microgrid in islanded and grid-connected modes," IEEE Trans. Power Syst., vol. 25, no. 4, pp. 1883-1891, 2010. 
[9] F. Guo, C. Wen, J. Mao, and Y. D. Song, "Distributed Secondary Voltage and Frequency Restoration Control of Droop-Controlled Inverter-Based Microgrids," IEEE Trans. Ind. Electron., vol. 62, no. 7, pp. 4355-4364, 2015.

[10]S. Xia, S. Bu, C. Wan, X. Lu, K. W. Chan, and B. Zhou, "A Fully Distributed Hierarchical Control Framework for Coordinated Operation of DERs in Active Distribution Power Networks," IEEE Trans. Power Syst., vol. 34, no. 6, pp. 5184-5197, 2019.

[11]H. Pourbabak, J. Luo, T. Chen, and W. Su, "A novel consensus-based distributed algorithm for economic dispatch based on local estimation of power mismatch," IEEE Trans. Smart Grid, vol. 9, no. 6, pp. 5930-5942, Nov. 2018.

[12] Y. Wang, L. Wu, and S. Wang, "A Fully-Decentralized Consensus-Based ADMM Approach for DC-OPF with Demand Response," IEEE Trans. Smart Grid, vol. 8, no. 6, pp. 2637-2647, Nov. 2017.

[13]D. Yang, S. Member, S. Zhang, and B. Zhou, "Consensus-Based Decentralized Optimization for Distributed Generators Power Allocation Over Time-Varying Digraphs in Microgrids," IEEE Syst. J., pp. 1-12, 2020.

[14]F. Mehmood, B. Khan, S. M. Ali, B. Qureshi, C. Diver, and R. Nawaz, "Multi-Renewable Energy Agent Based Control for Economic Dispatch and Frequency Regulation of Autonomous Renewable Grid," IEEE Access, vol. 8, pp. 1-1, 2020.

[15] Y. Liu, L. Xiao, G. Yao, and S. Bu, "Pricing-Based Demand Response for a Smart Home with Various Types of Household Appliances Considering Customer Satisfaction," IEEE Access, vol. 7, pp. 86463-86472, 2019.

[16]L. Xiao and S. Boyd, "Fast linear iterations for distributed averaging," Syst. Control Lett., vol. 53, no. 1, pp. 65-78, 2004.

[17]Z. Zhang and M. Y. Chow, "Convergence analysis of the incremental cost consensus algorithm under different communication network topologies in a smart grid," IEEE Trans. Power Syst., vol. 27, no. 4, pp. 1761-1768, 2012.

[18]N. F. Avila and C. C. Chu, "Distributed Pinning Droop Control in Isolated AC Microgrids," IEEE Trans. Ind. Appl., vol. 53, no. 4, pp. 3237-3249, 2017.

[19]A. Di Meglio, P. De Lellis, and M. Di Bernardo, "Decentralized gain adaptation for optimal pinning controllability of complex networks," IEEE Control Syst. Lett., vol. 4, no. 1, pp. 253-258, 2020.

[20] S. Manaffam, M. Talebi, A. K. Jain, and A. Behal, "Intelligent Pinning Based Cooperative Secondary Control of Distributed Generators for Microgrid in Islanding Operation Mode," IEEE Trans. Power Syst., vol. 33, no. 2, pp. 1364-1373, 2018.

[21] A. Bidram, A. Davoudi, F. L. Lewis, and J. M. Guerrero, "Distributed cooperative secondary control of microgrids using feedback linearization," IEEE Trans. Power Syst., vol. 28, no. 3, pp. 3462-3470, 2013. 